\title{
Parametric Effect on Surface Finish of Three-Dimensional Printed Object
}

\author{
Muhammad Hasibul Hasan, Shugata Ahmed, Ron Voldman and Mirt Mehany
}

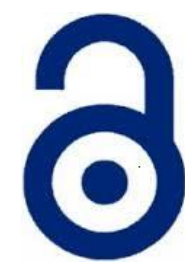

\author{
Received: 20 April 2018 \\ Accepted: 29 May 2018 \\ Published: 30 June 2018 \\ Publisher: Deer Hill Publications \\ (c) 2018 The Author(s) \\ Creative Commons: CC BY 4.0
}

\begin{abstract}
The ability to use different parameters and finishing techniques in fused deposition modelling (FDM) depends largely on part geometries, materials, printing processes like Z-resolution and post processing to some extent. Low quality poor surface finish due to layer ovality, improper Z-resolution parameter selection and fill of the empty shell in threedimensional (3D) printing, results unexpected texture and appearance. An investigation is carried out on the effects of Z-resolution $(0.15 \mathrm{~mm}$ to $0.40 \mathrm{~mm}$ ) and Fill parameters on flat and curved surface objects manufactured using the FDM process. Moreover, post surface treatment was performed using acetone. It was found that average surface roughness increases with increasing Z-resolution. Solid Fill can create a smoother surface as oppose to Sparse Fill. Surface roughness improves significantly after post treatment with organic solvent acetone.
\end{abstract}

Keywords. Fused deposition modelling, 3D printing, Z- resolution, Surface roughness

\section{INTRODUCTION}

Three-dimensional (3D) printers are used to fabricate rapid prototypes (RP) for research and analysis purposes. Besides rapid prototyping, 3D printing is also used for rapid manufacturing - a new manufacturing technique where 3D printers are used for short run custom manufacturing. In this method, printed objects are not prototypes but actual end user products. The basic principle of fused deposition modelling (FDM), 3D printing or additive manufacturing is to manufacture a prototype layer-by-layer at which most of the printing parameters can be adjusted. Surface finish is a major concern in FDM process, and as better surface finish is highly recommended for rapid prototypes and rapid manufacturing products, a large number of research has been carried out on it. In FDM, surface finish can be improved by three methods - $Z$ resolution, fill of filament and post treatment.

The literature has revealed that Fill parameter affects the surface finish - Fill is the material used to fill the empty space inside the shell of an object. Therefore, in order to achieve a better surface finish, the correct type of Fill should be used, and choosing a Fill pattern depends on the kind of model, desired structural strength and printing speed. A parametric study on the fabrication of a specimen using the FDM 3000 3D printer was conducted by Galantucci et al. (2015) The specimen material in use were ABS resins and PLA, in which specimens were fabricated by the hot extrusion technique and the objective was to improve the specimen's dimensional accuracy. Optimized parameters were determined by the factorial analysis design of the experiment (DOE). However, it was observed that after depositing the material, shrinkage occurred due to the large temperature difference $\left(217^{\circ} \mathrm{C}\right)$ between deposited material and deposition platform. For this reason, a deviation from ideal dimensions was observed.

Guerrero-de-Mier et al. (2015) focused on reducing warping and deformation due to internal stress occurring in FDM. For experimental purposes, hexagonal and square shape bricks were used as test parts. A G code was developed to fabricate the brick stacks, which were locked spatially. Authors suggested that warping can be reduced by limiting stacking section length. Akande (2015) claimed that low layer thickness, low speed of depositions and low Fill density are the optimal parameters to have for a better surface roughness. Khan et al. (2006) investigated the emphasis of slice thickness and support parameters on surface roughness - they mentioned that lower slice thickness produces

\footnotetext{
M. H. Hasan ${ }^{1}$, S. Ahmed ${ }^{2}$, R. Voldman ${ }^{3}$ and M. Mehany ${ }^{4}$

1,3,4Department of Mechanical and Industrial Engineering

Ryerson University, Canada

2Department of Robotics and Mechatronics Engineering

University of Dhaka, Dhaka 1000, Bangladesh

'E-mail: hasibulhasan@ryerson.ca, 2E-mail: shugataahmed.rme@du.ac.bd

3E-mail: rvoldman@ryerson.ca, ${ }^{4} \mathrm{E}-$-mail: mirt.mehany@ryerson.ca
}

Reference: Hasan, M. H., Ahmed, S., Voldman, R. and Mehany, M. (2018). Parametric Effect on Surface Finish of Three-Dimensional Printed Object. International Journal of Engineering Materials and Manufacture, 3(2), 98-104. 
better surface finish. Bakar et al. (2010) found optimal parameters for dimensional accuracy, better surface finish and reduced post-processing time.

According to Galantucci et al. (2009), chemical treatment is fast, cheap, and easy to use, which significantly improves surface finish. In their study, Percoco et al. (2012) investigated the effect of chemical treatment on compressive strength and surface roughness of FDM parts. For their chemical treatment, they used a solution composed of $90 \%$ dimethyl ketone and 10\% water, and they had found that the compressive strength of the parts was improved in this method. Researchers concluded that the proposed finishing treatment can be used with immersion time up to 300 sec to reduce roughness up to $90 \%$, which in addition to compressive strength, improves mechanical properties as well. Vargas-Alfredo et al. (2018) suggested that surface topography and chemical composition in a 3D printed porous object can be improved by immersing the object in polystyrene solution (PS). Fused deposition modelling (FDM) technique was utilized to fabricate the 3D parts with different shapes (screws, honeycombs, tubes and scaffolds). 400 $\mathrm{nm}$ to $3 \mu \mathrm{m}$ size pores were created on the surface of the 3D objects by immersing them in the PS solution - this method is known as the Breath Figures approach. Temperature, relative humidity and polymer concentration of the solution and time of immersion have significant effects on the pore size, for when the object was immersed in the solution for $1 \mathrm{~s}, 1.7 \pm 0.4 \mu \mathrm{m}$ pores were created. For $3 \mathrm{~s}$ and $5 \mathrm{~s}$ immersion times, $1.9 \pm 0.5 \mu \mathrm{m}$ and $2.4 \pm 0.4 \mu \mathrm{m}$ pores were created on the surface, respectively. BF method was used for chemical modification.

Rao et al. (2012) discussed that the surface finish of FDM parts can be improved by interacting the surface with vapours of tetrahydrofuran. In this process, the part to be smoothed out is placed on a non-soluble support inside a closed vessel with a non-air-tight lid. Heat is then applied to evaporate the tetrahydrofuran so that it can interact with the object's surface to make it smooth. Ahn et al. (2004) performed post processing to increase optical transmissivity by applying elevated temperatures and by providing resin infiltration and surface sanding. Pandey et al. (2003) examined the use of hot cutter machining to improve surface finish of FDM parts and it was concluded that the proposed machining method was able to produce the surface finish of 0.3 microns, which can be used to obtain a better surface finish of an FDM part. Sanatgar et al. (2017) carried out an investigation on 3D printing by deposition of polymers on synthetic fabrics. Fused deposition modelling (FDM) technique was used for adhesion. Effect of 3D printing process parameters such as extruder temperature, platform temperature and printing speed on the adhesion process were all investigated. It was found that effect of the extruder temperature on adhesion is linear. However, linear effect of platform temperature on adhesion process was insignificant. Effect of fabric and filler type on adhesion has been found to be significant. The purpose of this study was to investigate how to improve surface finish of FDM 3D printing by focusing on Fill and Z-Resolution parameters. Surface finish was also examined after post treatment by acetone.

\section{EXPERIMENT}

The UP plus 3D printer with UP plus software was used to print the specimens and Acrylonitrile Butadiene Styrene (ABS) was used as the object material. Printed samples are shown in Figure 1 and different surfaces of a sample are shown in Figure 2. Z-resolution were maintained between $0.15 \mathrm{~mm}$ to $0.40 \mathrm{~mm}$ even though the smallest particle height $0.10 \mathrm{~mm}$ or 100 microns could be achieved. The stepper motor of the printer can move the platform by as little as 100 microns, however FDM extruders cannot control the flow of filament precisely enough to produce clean results. For this reason, low micron prints on FDM machines often end up looking worse than high micron prints even though the individual layers may be finer.

The modelling envelope temperature is regulated to aid in the bonding process. The FDM head deposits material as it follows the part geometry that has been constructed from bottom and builds up the model to the top in SolidWorks 2013. Starting from the STL file, the geometry of a part can be read by the UP Plus software. Objects are printed for different Z-resolutions and Fills. Various Z-resolutions and Fills with relevant sample numbers are mentioned in Table 1, and after printing, $R_{a}$ values were measured by surface measuring instrument Surftest SV.500 of Mitutoyo. Images of surface texture on specimens were captured by a metallurgical microscope - Nikon Epiphot 200 and a sample image is given in Figure 3.

Post treatment was carried out to improve the surface finish of the specimen using acetone. The specimen was placed on a non-soluble support of aluminium foil raft inside a closed vessel with a non-air-tight lid. Heat was applied to evaporate the acetone for 15 to 20 minutes, so that it can interact with the object's surface to make the surface smooth. After post treatment, $R_{a}$ values were measured again. In 3D printing, using thinner layers has few to no advantages and only serves to increase print time. Thinner layers are most useful for improving the surface finish on parts that have diagonal or curved surfaces, such as surface 2 in the study (Figure $2 b$.)
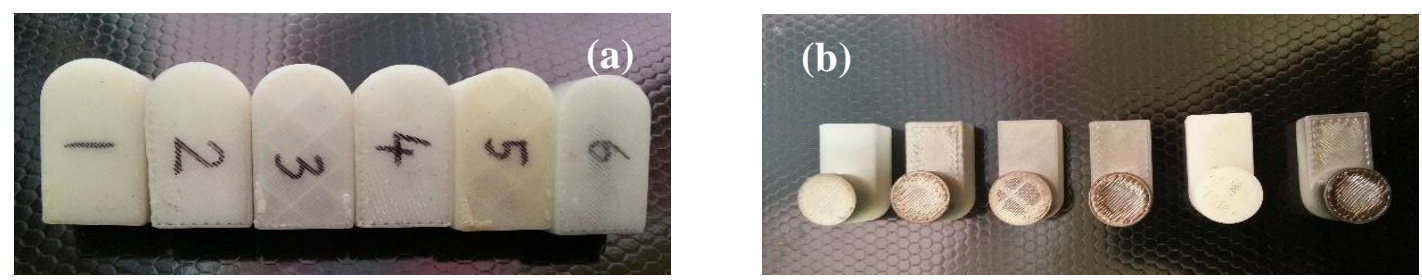

Figure 1: The printed samples 
Table 1: Types of Fill and Z-Resolution parameters applied on samples

\begin{tabular}{ccc}
\hline No. of samples & Z-Resolution $(\mathrm{mm})$ & Fill \\
\hline 1 & 0.15 & Solid \\
2 & 0.40 & Solid \\
3 & 0.15 & Sparse \\
4 & 0.40 & Sparse \\
5 & 0.15 & Sparse \\
6 & 0.40 & Sparse \\
\hline
\end{tabular}
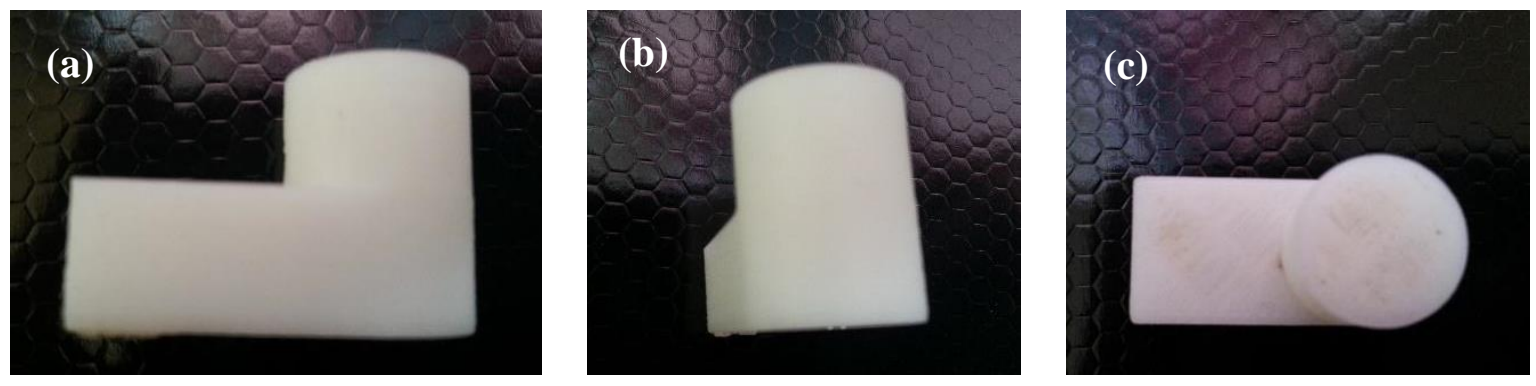

Figure 2: The view for surface 1 (a), surface 2 (b) and surface 3 (c) used in this study

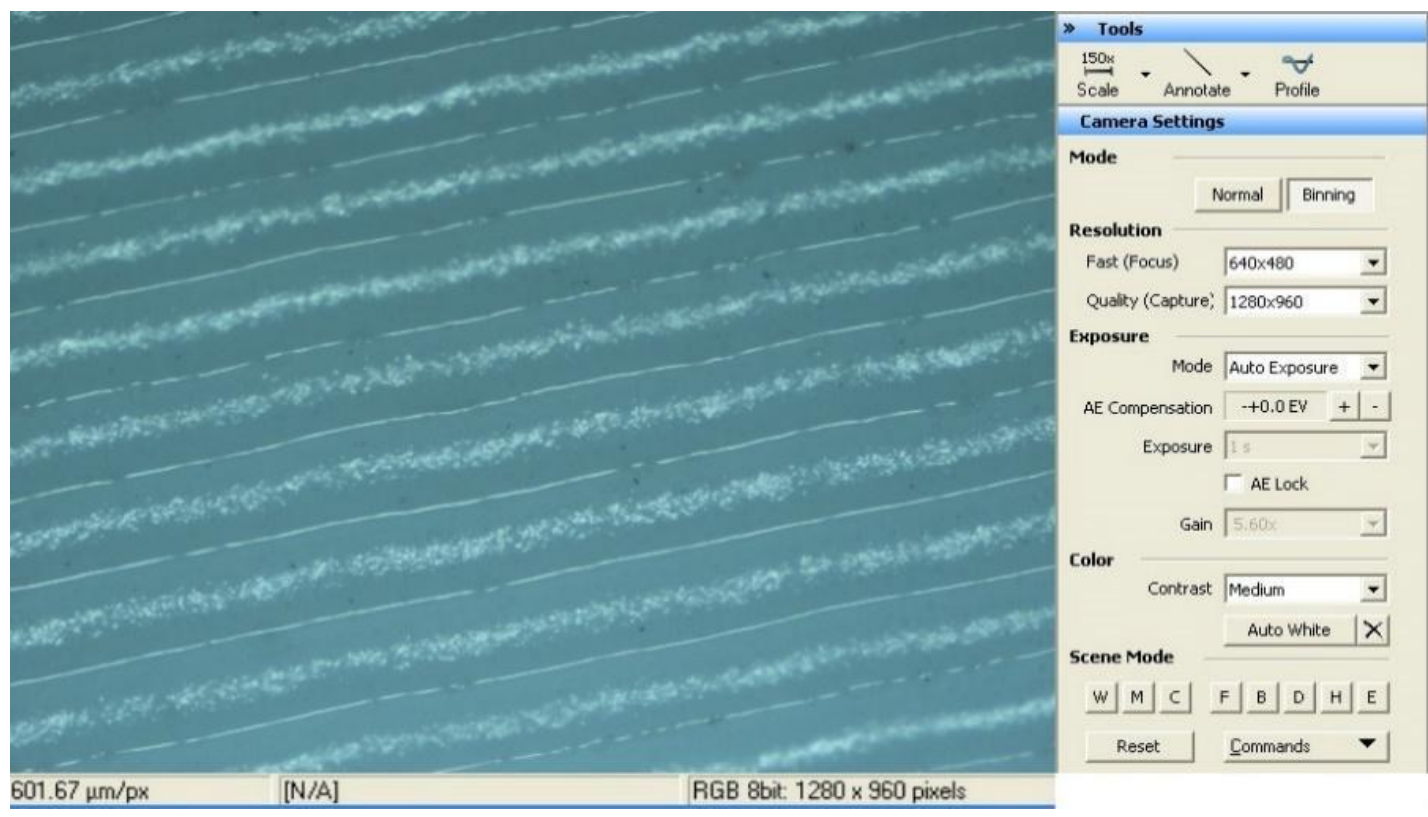

Figure 3: An image of the flat surface for sample 1 taken by microscope with magnification factor of 50X

\section{RESULTS AND DISCUSSIONS}

Average surface roughness was calculated for all six samples on their three different surfaces and two filling methods, as shown in the Table 2. From the Table 2, it can be seen that sample 1 had the lowest value of average surface roughness for surface 1, 2 and 3, which were $12.46 \mu \mathrm{m}, 12.27 \mu \mathrm{m}$ and $7.10 \mu \mathrm{m}$, respectively. Meanwhile, sample 4 had the highest value, which were $31.43 \mu \mathrm{m}, 28.49 \mu \mathrm{m}$, and the third surface was out of range due to the higher value of surface roughness. Sample 3 had a lower value of surface roughness for surface 1 and 2 compared to sample 2 , which were $15.85 \mu \mathrm{m}$ and $12.38 \mu \mathrm{m}$, respectively. However, sample 2 had a lower value for surface 3 of 24.62 $\mu \mathrm{m}$ compared to sample 3 , which was $36.52 \mu \mathrm{m}$. Hence, it is inferred that sample 1 had a better surface finish than 
other samples as it possessed the lowest average surface roughness values. It is interesting to note that a solid fill with higher Z-resolution produces a somewhat better surface finish despite a higher printing time.

Figure 4 shows the rising trend of the average surface roughness value with the increment of Z-resolution. From the figure, it is observed that surface 3 had the lowest value of average surface roughness $(7.10 \mu \mathrm{m})$ for a $0.15 \mathrm{~mm}$ Z-resolution. On the other hand, surface 1 had the highest surface roughness value of $31.54 \mu \mathrm{m}$ for a $0.4 \mathrm{~mm} \mathrm{Z-}$ resolution. In comparison, surface 3 had a better surface finish than other two surfaces for solid Fill parameter since it has the lowest roughness value for $0.15 \mathrm{~mm}$ and $0.275 \mathrm{~mm}$ of Z-resolutions. However, for a $0.4 \mathrm{~mm}$ Z-resolution, surface 2 had the lowest average surface roughness of $24.52 \mu \mathrm{m}$.

Table 2: The value of surface roughness for each surfaces of each samples

\begin{tabular}{|c|c|c|c|c|c|}
\hline & & \multicolumn{3}{|c|}{$\mathrm{R}_{\mathrm{a}}$ Value $(\mu \mathrm{m})$} & $\begin{array}{l}\text { Average } R_{a} \\
\text { Value }(\mu \mathrm{m})\end{array}$ \\
\hline SAMPLE No. & SURFACE No. & 1 & 2 & 3 & \\
\hline \multirow[t]{3}{*}{ 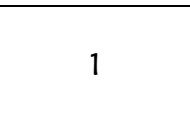 } & 1 & 12.40 & 12.67 & 12.31 & 12.46 \\
\hline & 2 & 12.20 & 12.39 & 12.23 & 12.27 \\
\hline & 3 & 7.11 & 7.11 & 7.09 & 7.10 \\
\hline \multirow{3}{*}{2} & 1 & 31.55 & 31.54 & 31.52 & 31.54 \\
\hline & 2 & 24.53 & 24.51 & 24.51 & 24.52 \\
\hline & 3 & 24.62 & 24.63 & 24.61 & 24.62 \\
\hline \multirow{3}{*}{3} & 1 & 15.85 & 15.86 & 15.84 & 15.85 \\
\hline & 2 & 12.41 & 12.39 & 12.35 & 12.38 \\
\hline & 3 & 36.44 & 36.63 & 36.49 & 36.52 \\
\hline \multirow{3}{*}{4} & 1 & 31.39 & 31.46 & 31.44 & 31.43 \\
\hline & 2 & 28.52 & 28.46 & 28.48 & 28.49 \\
\hline & 3 & \multicolumn{3}{|c|}{ Out of range } & \\
\hline \multirow{3}{*}{5} & 1 & 15.65 & 16.66 & 15.68 & 16.00 \\
\hline & 2 & 12.53 & 12.50 & 12.48 & 12.50 \\
\hline & 3 & 36.42 & 36.48 & 36.45 & 36.45 \\
\hline \multirow{3}{*}{6} & 1 & 1.56 & 1.69 & 1.57 & 1.61 \\
\hline & 2 & 0.84 & 0.92 & 0.90 & 0.89 \\
\hline & 3 & \multicolumn{3}{|c|}{ Out of range } & \\
\hline
\end{tabular}

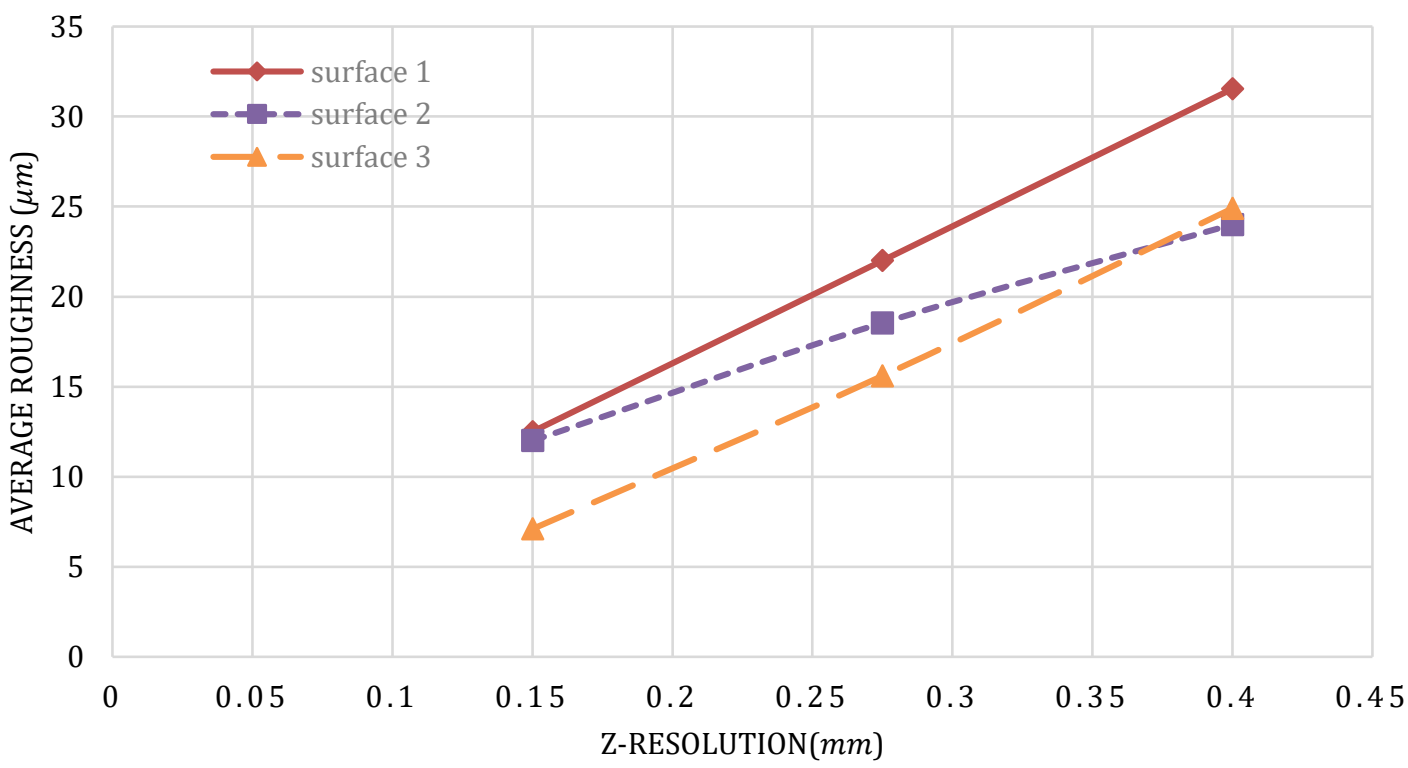

Figure 4: The $R_{a}$ value depending on Z-resolution of two Solid Fill samples 
Similarly, for sparse Fill, better surface finish was found for surface 2 in Figure 5. On the other hand, surface 3 showed the highest average roughness for sparse Fill. As the Z-resolution increased from $0.15 \mathrm{~mm}$ to $0.45 \mathrm{~mm}$, the surface roughness increased relatively linearly - reducing the 3D printing time for sparse fill samples.

Figure 6 shows the trend of average surface roughness with changing Fill parameters. From the figure, it can be seen that the average roughness value for surface 2 and 3 increased as it went from solid to sparse Fill parameter. However, there was no value for average roughness of surface 3 with sparse Fill parameter, as the reading was out of range. Meanwhile, surface 1 displayed a slightly downward trend when the sample was filled with the sparse Fill parameter. Nevertheless, its average surface roughness was the highest between the other two surfaces.

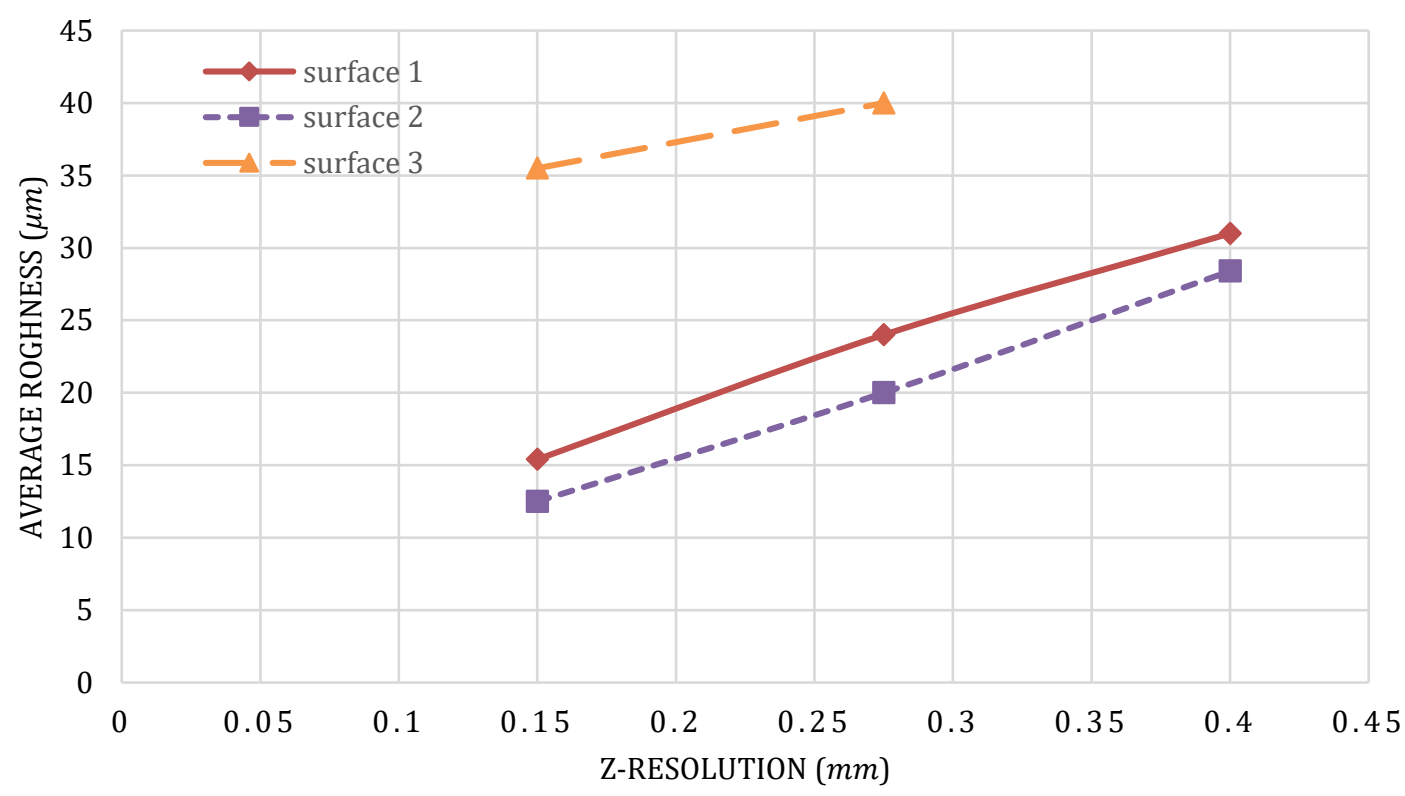

Figure 5: The $R_{a}$ value depending on Z-resolution of two sparse Fill samples

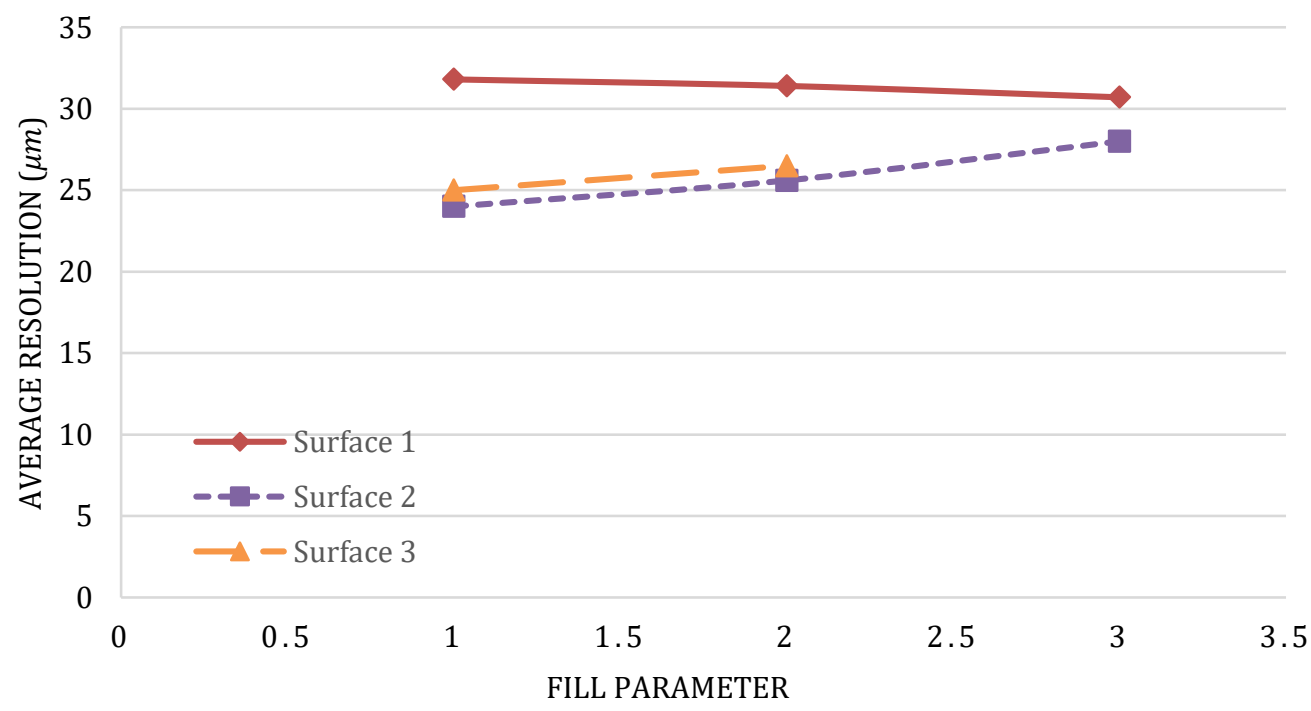

Figure 6: The $R_{a}$ value depending on Fill parameter of two $0.40 \mathrm{~mm}$ Z-Resolution samples 


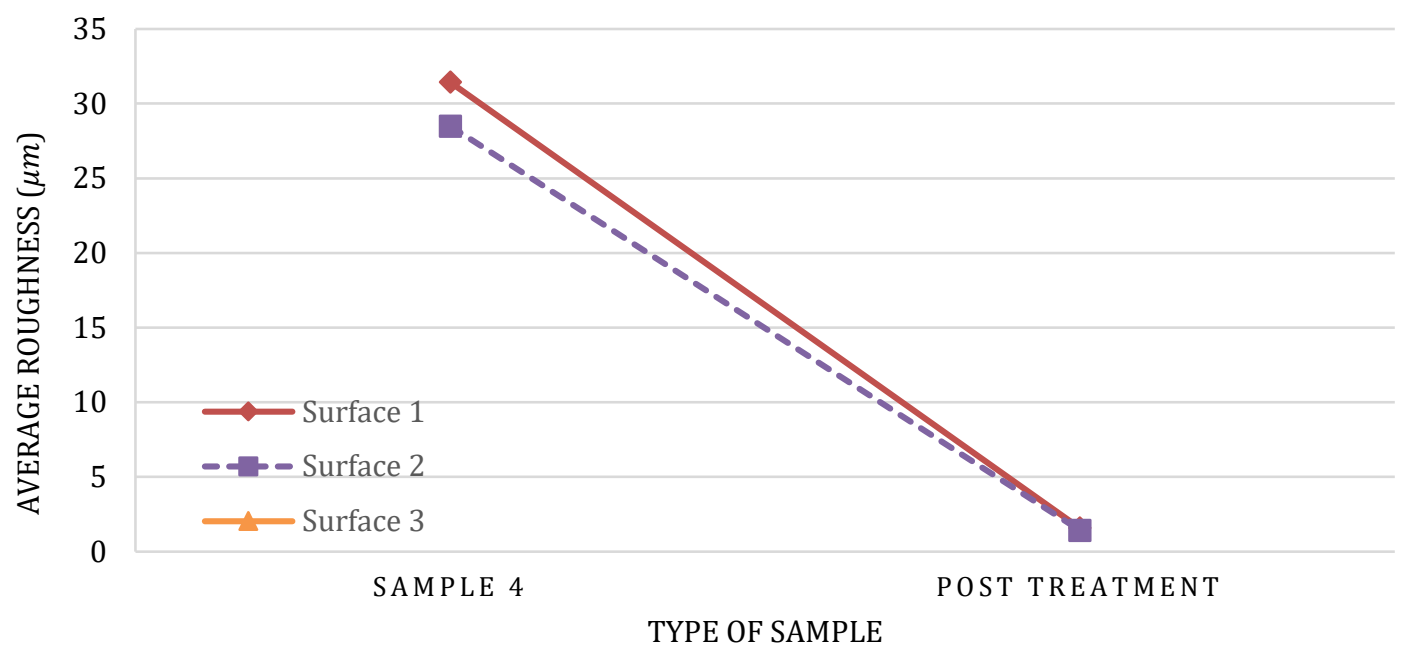

Figure 7: Comparison of surface roughness between untreated sample and sample after post treatment

In Figure 7, it is observed that surface roughness of surface 1 and surface 2 of sample 4 were $31.43 \mu \mathrm{m}$ and 28.49 $\mu \mathrm{m}$, respectively, before post treatment. However, after post treatment - the lowest values of surface roughness for both surfaces were achieved. This result supports a study conducted by Lalehpour et al. where it was experimentally proved that chemical finishing processes in FDM, can meet the conditions of a functional and adequate finishing process, as it improves the roughness without negatively affecting the mechanical or geometrical properties of the treated parts. [13]. Lalehpour et al. (2018) showed that the maximum reduction percentage of the surface roughness due to the treatment reaches $95 \%$ and no effect was observed on the build orientation, dimensional accuracy and dimensional deviation.

\section{CONCLUSIONS}

The carried out experiment studies the effect of different parameters on surface roughness of 3D printed objects manufactured by the FDM process. The following key factors are determined from the experimental results:

1. Average surface roughness is high for higher values of Z-resolution for a flat surface. For a curved surface, the $Z$-resolution effect is more pronounced. It is recommended to use a higher resolution or a lower $Z$ value for better curved surfaces at the expense of a longer printing time.

2. Solid Fill is better than Sparse Fill for generating smoother surfaces, even though more materials would be consumed in making the sample heavy.

3. Smoother surface was developed after post treatment by organic compound acetone, which can meet the conditions of a functional and adequate finishing process, as it improves the roughness without affecting the mechanical or geometrical properties of the treated parts. No effect was observed on the build orientation, dimensional accuracy and dimensional deviation.

\section{REFERENCES}

1. Guerrero-de-Mier, A., Espinosa, M. M., \& Domínguez, M. (2015). Bricking: A new slicing method to reduce warping. Procedia Engineering, 132, 126-131.

2. Galantucci, L. M., Bodi, I., Kacani, J., \& Lavecchia, F. (2015). Analysis of dimensional performance for a 3D open-source printer based on fused deposition modeling technique. Procedia CIRP, 28, 82-87.

3. Akande, S. O. (2015). Dimensional accuracy and surface finish optimization of fused deposition modeling parts using desirability function analysis. International Journal of Engineering Research \& Technology, 4(4), 196-202.

4. Khan, S. F., Salleh A. F, Sharif, S., Zulkepli M., Zulkifli, A. R., \& Nasir, M. M. (2006) A preliminary study on FDM prototype surface roughness. Proceeding of Malaysian Technical Universities Conference Engineering and Technology, MUCET. 
5. Bakar, N.S.A., Alkahari, M.R. \& Boejang, H. (2010). Analysis on fused deposition modelling performance. Journal of Zhejiang University-SCIENCE A (Applied Physics \& Engineering), 11-12, 972-977.

6. Galantucci, L.M., Lavecchia, F. \& Percoco, G. (2009). Experimental study aiming to enhance the surface finish of fused deposition modeled parts. CIRP Annals - Manufacturing Technology, 58(1), 189-192.

7. Percoco, G., Lavecchia, F. \& Galantucci, L. M. (2012). Compressive properties of FDM rapid prototypes treated with a low cost chemical finishing. Research Journal of Applied Sciences, Engineering and Technology, 19(4), 3838-3842.

8. Vargas-Alfredo, N., Reinecke, H., Gallardo, A., del Campo, A., \& Rodríguez-Hernández, J. (2018). Fabrication of 3D printed objects with controlled surface chemistry and topography. European Polymer Journal, 98, 21-27.

9. Rao, A.S., Dharap, M.A.,Venkatesh, J.V.L. \& Ojha, D. (2012). Investigation of post processing techniques to reduce the surface roughness of fused deposition modeled parts. International Journal Of Mechanical Engineering and Technology, 3(3), 531-544.

10. Ahn, S., Lee, C., \& Jeong, W. (2004). Development of translucent FDM parts by post-processing. Rapid prototyping Journal, 10 (4), 218-224.

11. Pandey, P. M., Reddy, N. V. \& Dhande, S. G.(2003). Improvement of surface finish by staircase machining in fused deposition modelling. Journal of Materials Processing Technology, 132, 323-331.

12. Sanatgar, R. H., Campagne, C., \& Nierstrasz, V. (2017). Investigation of the adhesion properties of direct 3D printing of polymers and nanocomposites on textiles: Effect of FDM printing process parameters. Applied Surface Science, 403, 551-563.

13. Gajdos, I., Spisak, E., Kascak, L., \& Krasinskyi, V. (2015). Surface finish techniques for FDM parts. Materials Science Forum, 818, 45-48. doi: https://doi.org/10.4028/www.scientific.net/MSF.818.45. 